\title{
Heat bath can generate all classes of three-qubit entanglement
}

\author{
Christopher Eltschka, ${ }^{1}$ Daniel Braun, ${ }^{2,3,4}$ and Jens Siewert ${ }^{5,6}$ \\ ${ }^{1}$ Institut für Theoretische Physik, Universität Regensburg, D-93040 Regensburg, Germany \\ ${ }^{2}$ Laboratoire de Physique Théorique - Université de Toulouse Paul Sabatier, F-31062 Toulouse, France \\ ${ }^{3}$ Laboratoire de Physique Théorique - Insitut de Recherche sur les Systèmes Atomiques et Moléculaires Comples, Centre National de la \\ Recherche Scientifique (CNRS), F-31062 Toulouse, France \\ ${ }^{4}$ Institut für theoretische Physik, Universität Tübingen, D-72076 Tübingen, Germany \\ ${ }^{5}$ Departamento de Química Física, Universidad del País Vasco UPV/EHU, 48080 Bilbao, Spain \\ ${ }^{6}$ IKERBASQUE, Basque Foundation for Science, 48011 Bilbao, Spain
}

(Received 6 January 2014; published 6 June 2014)

\begin{abstract}
It is common knowledge that coupling to a heat bath, in general, tends to reduce the entanglement in a quantum system. In recent years, increasing interest has been devoted to the opposite situation where thermal or specifically tailored environments may stabilize or even generate entanglement. We prove the universality of this phenomenon for multipartite entanglement in the frame of an exactly solvable dephasing model. We show by evaluating analytical lower bounds for the appropriate entanglement measures that a common thermal environment can dynamically generate all entanglement classes of three qubits for almost any initial product state. For sufficiently weak dissipation this entanglement may persist up to arbitrarily large times.
\end{abstract}

DOI: 10.1103/PhysRevA.89.062307

PACS number(s): 03.67.Bg, 03.65.Yz, 05.70.-a

\section{INTRODUCTION}

Entanglement, one of the hallmarks of quantum mechanics with a wide range of applications in quantum information processing [1], is commonly thought of as being rather fragile when exposed to decohering environments. Yet, already towards the end of the last century, it turned out that dissipative dynamics can lead to entanglement due to relaxation rates that depend on the multipartite state [2], stabilize entanglement in a decoherence-free subspace [3], or enable its generation through local external driving [4,5]. Later it was shown theoretically that specific environments can be used to engineer highly entangled states [6,7], with very recent experimental implementations [8,9]. Apart from specifically tailoring dissipation it is interesting to ask about the action of a more generic or even thermal environment. The somewhat surprising answer is that the interaction with a common heat bath alone can, under certain circumstances, entangle two subsystems [10]. This kind of "reservoir-induced entanglement" has meanwhile been found for a large variety of Markovian and non-Markovian evolutions, systems with finite- or infinite-dimensional Hilbert spaces, many different environments (spins, bosons, squeezed or thermal, single and many mode), Brownian motion, and even vacuum or the Unruh radiation experienced by accelerated subsystems [11-18].

The physical picture behind this effect is that the common heat bath not only leads to decoherence, but also to an effective interaction. Depending on the balance between the two, entanglement may arise, disappear again, or even persist in a steady state. With very few exceptions [6,7,19-23] these investigations were all concerned with bipartite entanglement. However, entanglement in systems with more than two parties exhibits a rich structure that is little explored. Three qubits represent the only multipartite system whose state space partitioning in terms of entanglement classes is completely known [24,25]. It is known, however, that multipartite entanglement is rather fragile under local decoherence, and there are no states that would provide maximum robustness against all decoherence channels in the set of phase damping, depolarizing, bit flip, phase flip, and bit-phase flip. The situation for three qubits is particularly complex in this respect [26]. Thus the question arises as to what extent reservoir-induced entanglement generation is a universal phenomenon and whether all entanglement types in a multipartite system can be generated from separable states. The latest developments in entanglement theory have made it feasible to give a quantitative answer.

Here we examine the generation of genuine tripartite entanglement for three qubits in a large class of thermal dephasing environments using state-of-the-art methods to detect the various entanglement resources. We show that all entanglement classes can arise from the coupling to the common heat bath alone and find the corresponding conditions for the model parameters and initial states. We start our discussion by introducing the dephasing models under consideration and solve the equations of motions exactly. Before discussing in detail the entanglement classes in the system evolution we briefly review the entanglement classification for three qubits and the appropriate measures to quantify these resources [27].

\section{MODEL}

Consider a system of qubits $H_{\text {sys }}$ interacting with a heat bath. We will restrict ourselves to noninteracting qubits with degenerate energy levels, i.e., $H_{\text {sys }}=0$, so that the total Hamiltonian reads

$$
H=H_{\text {int }}+H_{\text {bath }} .
$$

This type of model is also obtained from a more general dephasing model (i.e., a model with $\left[H_{\text {sys }}, H_{\text {int }}\right]=0$ ) by going to the interaction picture with respect to $H_{\text {sys }}$. The system is coupled through an interaction Hamiltonian $H_{\text {int }}=S \otimes B$ to a common heat bath, where the "system-coupling agent" $S$ acts on the qubit Hilbert space, and the "bath-coupling agent" $B$ acts on the bath degrees of freedom. For the heat bath we 
assume a set of harmonic oscillators,

$$
H_{\mathrm{bath}}=\sum_{j}\left(\frac{p_{j}^{2}}{2 m}+\frac{1}{2} m \omega_{j}^{2} q_{j}^{2}\right),
$$

and $B=\sum_{j} g_{j} q_{j}$ where $g_{j}$ are coupling constants to the $j$ th oscillator. It is most convenient to solve the dynamics of the resulting reduced density matrix in the pointer basis $\{|s\rangle\}$ with $S|s\rangle=s|s\rangle$. With the usual assumptions of factorizing initial conditions between system and bath, and the bath in thermal equilibrium at temperature $T=1 /\left(k_{B} \beta\right)$ at time $t=0$, one finds the exact general solution in terms of the reduced density matrix $\rho(t)$ of the von Neumann equation of motion [28]:

$$
\left\langle s|\rho(t)| s^{\prime}\right\rangle=e^{-\left(s-s^{\prime}\right)^{2} f(t)+i\left(s^{2}-s^{\prime 2}\right) \varphi(t)}\left\langle s|\rho(0)| s^{\prime}\right\rangle,
$$

where the functions $f(t)$ and $\varphi(t)$ are, respectively, related to real and imaginary part of the thermal-bath correlation function:

$f(t)=\sum_{j} \frac{g_{j}^{2}\left(1+2 \bar{n}_{j}\right)}{2 m \hbar \omega_{j}^{3}}\left(1-\cos \omega_{j} t\right)=\operatorname{Re} \int_{0}^{t} \frac{d t^{\prime} t^{\prime}}{\hbar^{2}} C\left(t-t^{\prime}\right)$, $\varphi(t)=\sum_{j} \frac{g_{j}^{2}}{2 m \hbar \omega_{j}^{2}}\left[t-\frac{\sin \omega_{j} t}{\omega_{j}}\right]=\operatorname{Im} \int_{0}^{t} \frac{d t^{\prime} t^{\prime}}{\hbar^{2}} C\left(t-t^{\prime}\right)$,

where $C(t)=\langle B(t) B(0)\rangle$ is evaluated in the initial thermal state of the heat bath, and $\bar{n}_{j}=1 /\left(e^{\beta \hbar \omega_{j}}-1\right)$. One may also rewrite these two functions in terms of a spectral density $J(\omega)=\pi \sum_{j} \frac{g_{j}^{2}}{2 m \omega_{j}} \delta\left(\omega-\omega_{j}\right)$, which in the limit of dense frequencies $\omega_{j}$ is taken as a continuous function. Both $f(t)$ and $\varphi(t)$ vanish at $t=0$. For $t>0$ they are real and positive. While $\varphi(t) \propto t$ for large times, the behavior of $f(t)$ for large times depends on the heat bath. If there is an ultraviolet cutoff, $f(t)$ may saturate at some finite value, whereas otherwise $f(t)$ may diverge. Thus, the time evolution arising from the action of a given heat bath is characterized by a certain path $(f(t), \varphi(t))$ in the quadrant $\mathbb{R}_{\geqslant 0} \times \mathbb{R}_{\geqslant 0}$ [see Fig. 1 for paths arising from the spectral densities $J(\omega)=A \omega^{s} \theta\left(\omega-\omega_{c}\right)$, where $s>0, \theta(\omega)$

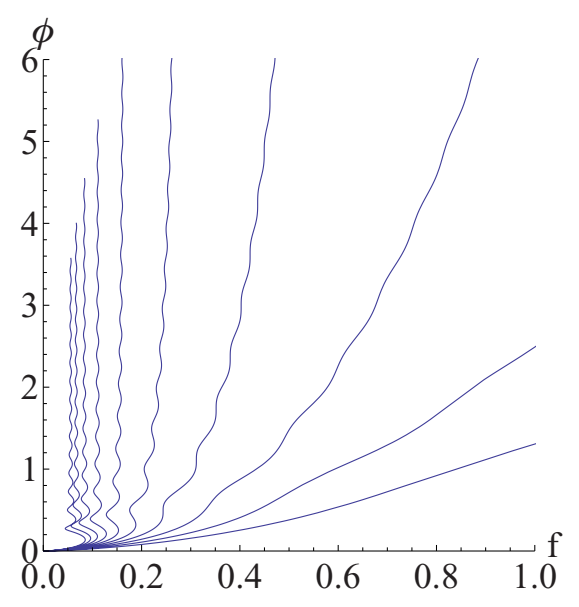

FIG. 1. (Color online) Examples of paths in the $f-\varphi$ plane for heat baths at temperature $T=0$ characterized by a spectral density $J(\omega)=A \omega^{v} \theta\left(\omega-\omega_{c}\right)$ for $v=0.1, \ldots, 2.8$ in steps of 0.3 from right to left, $A \omega_{c}^{\nu-1} /(\pi \hbar)=0.1$, and $0 \leqslant \tau=\omega_{c} t \leqslant 100$. is the Heaviside function, and $\omega_{c}$ a cutoff frequency]. It is worthwhile noting that the following results on entanglement evolution are applicable not only to the original dephasing model but also to any composite system with a reduced system density matrix which can be parameterized in the form (3), independent of the particular form (and origin) of the functions $f$ and $\varphi$.

\section{THREE QUBITS COUPLED TO A COMMON HEAT BATH}

\section{A. Parametrization of coupling Hamiltonian}

In the appropriate local bases the most general form of $S$ reads

$$
S=i \lambda_{0} \sigma_{0}^{(1)} \otimes \sigma_{0}^{(2)} \otimes \sigma_{0}^{(3)}+\sum_{j} \lambda_{j} \sigma_{z}^{(j)},
$$

where $\sigma_{\alpha}^{(j)}$ denotes the Pauli matrices for the $j$ th qubit ( $\alpha \in$ $\{x, y, z\}, j \in\{1,2,3\}), \sigma_{0}^{(j)} \equiv i \mathbb{1}$, and $\lambda_{j} \geqslant 0$. We assume $\lambda_{0}=$ 0 because a finite $\lambda_{0}$ amounts to shifting the bath oscillators and does not lead to qualitatively new behavior. Also, because of the symmetry of the problem, we may assume $\lambda_{1} \geqslant \lambda_{2} \geqslant \lambda_{3}$. Finally, a common multiplier of the $\lambda_{j}$ can be absorbed in the coupling constants $g_{i}$ and thus just corresponds to rescaling of $f$ and $\varphi$, therefore we may choose $\lambda_{1}=1$. Thus the general form of $S$ we use is

$$
S=\sigma_{z}^{(1)}+\lambda_{2} \sigma_{z}^{(2)}+\lambda_{3} \sigma_{z}^{(3)}, \quad 1 \geqslant \lambda_{2} \geqslant \lambda_{1} \geqslant 0,
$$

with eigenvalues $s_{j k l}$

$$
\begin{aligned}
S|j k l\rangle & =s_{j k l}|j k l\rangle \\
& =\left[(-1)^{j}+(-1)^{k} \lambda_{2}+(-1)^{l} \lambda_{3}\right]|j k l\rangle,
\end{aligned}
$$

which, when substituted in Eq. (3), determine the time evolution of the reduced-system state.

\section{B. Quantifying entanglement resources}

For three qubits, there are three types (or classes) of entanglement: Greenberger-Horne-Zeilinger (GHZ) type, $W$ type and, biseparable (B) [24,25]. A mixed state is of a given entanglement class if it has at least one decomposition that contains only states of this class, but it has no decomposition with states only from "lower" classes. To date, there are no practicable methods to identify with certainty the entanglement type of arbitrary mixed states. However, since the entanglement classes form a hierarchy GHZ $\supset \supset \mathrm{B}$, it is meaningful to determine the class a state at least belongs to.

To identify GHZ-type entanglement, we use the three tangle, which is nonzero exactly for GHZ-type entangled states $[29,30]$. For pure three-qubit states $\psi$ it is defined as (we drop the qubit index of the Pauli matrices)

$$
\tau_{3}=\sqrt{\left|\sum_{j=0, x, z}\left\langle\psi^{*}\left|\sigma_{j} \otimes \sigma_{y} \otimes \sigma_{y}\right| \psi\right\rangle\left\langle\psi^{*}\left|\sigma_{j} \otimes \sigma_{y} \otimes \sigma_{y}\right| \psi\right\rangle\right|}
$$

(here $\psi^{*}$ denotes the state with complex conjugate coefficients) and for mixed states $\rho$ with pure-state decompositions 
$\rho=\sum p_{j}\left|\psi_{j}\right\rangle\left\langle\psi_{j}\right|$ it is the convex roof [31]

$$
\tau_{3}(\rho)=\min _{\text {all decomp. }} \sum p_{j} \tau_{3}\left(\psi_{j}\right) .
$$

While there is no known way to calculate the exact three-tangle of arbitrary mixed states, a lower bound can, in principle, be calculated analytically [32]. If this lower bound does not vanish, we know for sure the state is GHZ-type entangled.

As for three qubits there are only two classes of genuine multipartite entanglement (GME) the appropriate quantifier to measure the " $W$-ness" of a state is the GME concurrence [33]. To this end we need to consider the three bipartitions $\{1 \mid 23\}$, $\{2 \mid 13\}$, and $\{3 \mid 12\}$ of qubit 1 , qubit 2 , and qubit 3 . For pure states the GME concurrence is the minimum linear entropy among all possible bipartitions $\gamma_{i}=\left\{A_{i} \mid B_{i}\right\}$ of a state

$$
C_{\mathrm{GME}}(\psi)=\min _{\gamma_{i}} \sqrt{2\left[1-\operatorname{tr}\left(\rho_{A_{i}}^{2}\right)\right]},
$$

where $\rho_{A_{i}}$ is the reduced density matrix of party $A_{i}$ in the bipartition $\gamma_{i}$. For mixed states, $C_{\mathrm{GME}}$ is defined as the convex roof, in analogy with Eq. (7). For the explicit calculations we use the lower bound described in Ref. [33]. The procedure to calculate the bounds involves an optimization in which we maximize the three-tangle or the GME concurrence over local unitary transformations.

To determine whether the state is entangled at all we use the negativity [34]. That is, we consider again the bipartitions $\gamma_{i}$, but now each of them separately. The negativity for the bipartition $\gamma_{i}$ is $\mathcal{N}_{\gamma_{i}}(\rho)=\frac{1}{2}\left(\left\|\rho^{T_{A_{i}}}\right\|_{1}-1\right)$ where $\rho^{T_{A_{i}}}$ denotes the partial transpose with respect to party $A_{i}$ and $\|\bullet\|_{1}$ is the trace norm. Also $\mathcal{N}(\rho)$ can be regarded as a lower bound because it does not detect entangled states with a positive partial transpose. However, it can be computed easily. Note that the maximum value of $\mathcal{N}$ for a single-qubit partition is $\frac{1}{2}$. As the maxima of $\tau_{3}$ and $C_{\mathrm{GME}}$ equal 1 we plot $2 \mathcal{N}$.

\section{EQUIVALENCE OF EVOLUTIONS OF PURE PRODUCT STATES}

Because we are interested in possible entanglement generation we study the time evolution starting with a fully separable pure state, i.e., $|\psi\rangle=\left|\phi_{1}\right\rangle\left|\phi_{2}\right\rangle\left|\phi_{3}\right\rangle$ with $\left|\phi_{j}\right\rangle=$ $\alpha_{j}|0\rangle+\beta_{j}|1\rangle$. All product states can be obtained from the state $|+\rangle=\frac{1}{\sqrt{2}}(|0\rangle+|1\rangle)$ by the diagonal transformation $F=$ $\otimes_{j=1}^{3} F_{j}$ with $F_{j}=\operatorname{diag}\left(\alpha_{j}, \beta_{j}\right)$ which, except for the special cases $\left|\phi_{j}\right\rangle=|0\rangle$ and $\left|\phi_{j}\right\rangle=|1\rangle$, is invertible. This in turn means that all tripartite product states that contain neither $|0\rangle$ nor $|1\rangle$ as a factor are related to each other by diagonal $\operatorname{GL}(2, \mathbb{C})^{\otimes 3}$ transformations.

Since we consider diagonal couplings $S$ to the bath the time evolution commutes with all diagonal transformations. Thus, for any given $f$ and $\varphi$, the state $\rho\left(f, \varphi ; \phi_{1} \phi_{2} \phi_{3}\right)$ is related to the state $\rho(f, \varphi ;+++)$ by the same diagonal transformation as the state $\left|\phi_{1} \phi_{2} \phi_{3}\right\rangle$ to the state $|+++\rangle$. In particular, as long as the initial state does not contain a factor $|0\rangle$ or $|1\rangle$, for fixed $f$ and $\varphi$ all states $\rho\left(f, \varphi ; \phi_{1} \phi_{2} \phi_{3}\right)$ can be transformed into one another by a $\mathrm{GL}(2, \mathbb{C})^{\otimes 3}$ transformation and thus belong to the same entanglement class. Consequently, it suffices to look at $|+++\rangle$ to capture the behavior of (almost) all initial product states.
We may apply analogous reasoning to GHZ-type entanglement and exploit the properties of the three tangle under local transformations [30]. For arbitrary $\rho$ and arbitrary local transformations $G \in \mathrm{GL}(2, \mathbb{C})^{\otimes 3}$, we have $\tau_{3}\left(G \rho G^{\dagger}\right)=$ $|\operatorname{det}(G)|^{2} \tau_{3}(\rho)$. For a diagonal transformation $G$ which turns $|+++\rangle$ into the normalized state $\left|\phi_{1} \phi_{2} \phi_{3}\right\rangle$ we find $|\operatorname{det} G|^{2}=$ $4\left|\prod_{j=1}^{3} \alpha_{j} \beta_{j}\right|^{2} \leqslant 1$. Hence the state $\rho(f, \varphi ;+++)$ has the largest three tangle among all the states with the same $f$ and $\varphi$. For any other state, the three tangle can be obtained by multiplication with the appropriate determinant.

In the case where one of the factors of the initial state is $|0\rangle$ or $|1\rangle$, the transformation $F$ is basically a projection, so that the qubit does not evolve at all. Thus, the problem effectively reduces to the two-qubit case (or the one-qubit case if two of the factors are $|0\rangle$ or $|1\rangle$ ). Therefore, in what follows we will assume that the initial state is

$$
\rho(t=0) \equiv \rho_{0}=\pi_{+++} \longrightarrow\left\langle j k l\left|\rho_{0}\right| m n q\right\rangle=\frac{1}{8},
$$

with the abbreviation $|\psi\rangle\langle\psi| \equiv \pi_{\psi}$.

\section{ENTANGLEMENT EVOLUTION IN $f$ - $\varphi$ PLANE}

\section{A. Small values of $f$}

The evolution starts at $t=0$ with the pure product state $\pi_{+++}$and $f=\varphi=0$. While a state can never reach a point with $f=0$ but $\varphi \neq 0$, it is still worthwhile considering this case because, in principle, arbitrarily small values of $f$ are possible. Moreover, because the states at $f=0$ are pure, all entanglement measures (which are continuous) can be evaluated exactly. The three tangle for $f=0$ is

$\tau_{3}(f=0, \varphi)=\frac{1}{2} \sqrt{\left|c_{1} c_{2} c_{3}+i s_{1} s_{2} s_{3}-\left(c_{1}+c_{2}+c_{3}\right)+2\right|}$,

where $c_{j}=\cos \left(8 \lambda_{j} \varphi(t)\right), s_{j}=\sin \left(8 \lambda_{j} \varphi(t)\right), j=1,2,3$, and $\lambda_{1} \equiv 1$. For small $\varphi$, the leading term is $\tau_{3}(\psi(\varphi))=$ $8 \sqrt{2} \lambda_{2} \lambda_{3}|\varphi|^{3 / 2}+O\left(|\varphi|^{7 / 2}\right)$. This shows that a three tangle can be produced whenever both $\lambda_{2}$ and $\lambda_{3}$ are nonzero; that is, whenever all three qubits are coupled to the bath. Only if both $\lambda_{2}$ and $\lambda_{3}$ are rational is the $\varphi$ dependence periodic. In that case there exists a sequence of values $\varphi_{n}$ with vanishing initial three tangle $\tau_{3}\left(f=0, \varphi_{n}\right)=0$. For $\varphi_{n}$ there is no entanglement at all in the system ("zero lines" of entanglement for all $f$ values).

Upon increasing $f$ the off-diagonal elements decay according to Eq. (3) so that the states become more and more mixed and the entanglement measures decrease. For our method we always expect three tangle and GME concurrence to vanish at finite $f$ values. This is because the fidelity of the initial GHZ-entangled state decreases with growing $f$ while our method does not detect $\tau_{3}$ or $C_{\mathrm{GME}}$ for GHZ fidelities of the optimized state $<\frac{1}{2}$. Nonetheless, this shows that if, for a given heat bath, $f(t \rightarrow \infty)$ saturates at a small-enough value, both types of genuine multipartite entanglement may persist up to arbitrarily large times. The dynamics of bipartite entanglement will be discussed below. The typical behavior of the entanglement measures as functions of $f$ and $\varphi$ for generic coupling parameters resembles the one displayed in Fig. 2.

\section{B. Asymptotic states for $f \rightarrow \infty$}

It is useful to study the behavior for $f \rightarrow \infty$ because it reveals the special cases that need to be considered. Recall 


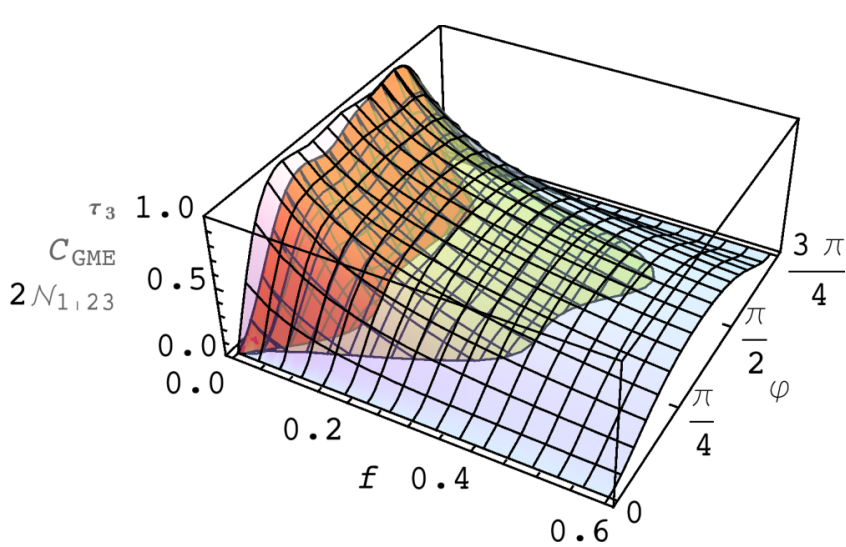

FIG. 2. (Color online) Entanglement classes GHZ, $W, \mathrm{~B}$ (red, yellow, light blue) for the case $\lambda_{2}=\frac{2}{3}, \lambda_{3}=\frac{1}{3}$. Qualitatively, the behavior resembles that of the generic case, only that here there is a periodicity in $\varphi$ with zero lines for the entanglement measures at the values of $\varphi=3 n \pi / 4$. The negativity is calculated for the bipartition $\{1 \mid 23\}$, i.e., for the first qubit.

that $f(t \rightarrow \infty)$ may diverge if not prevented by a cutoff. We conclude from Eq. (3) that $\rho(f \rightarrow \infty)=\sum_{r} P_{r} \rho_{0} P_{r}^{\dagger}$ where $P_{r}$ are the projectors on the eigenspaces of $S$. We have to determine those eigenspaces depending on $\lambda_{2}$ and $\lambda_{3}$. Since $S$ is diagonal in the computational basis, $|j k l\rangle$ are trivially eigenstates, with corresponding eigenvalues $s_{j k l}$ according to Eq. (6). Because we start with the pure state $|+++\rangle$, the asymptotic state $\rho(f \rightarrow \infty)$ is a mixture of pure states that are equally weighted superpositions of all the basis states in the corresponding eigenspace. Note that all entries of $\rho(f \rightarrow \infty)$ are either $\frac{1}{8}$ or 0 .

For generic values of $\lambda_{2}$ and $\lambda_{3}$, all eigenspaces are one dimensional, and $\rho(f \rightarrow \infty)$ is the completely mixed state. Since there is a finite neighborhood of the completely mixed state where all states are separable [35], generically full separability will be reached for finite values of $f$. However, for certain values of $\lambda_{2}$ and $\lambda_{3}$, some of the eigenvalues will coincide, resulting in larger eigenspaces and thus more interesting asymptotic states.

In the trivial case $\lambda_{2}=\lambda_{3}=0$ no entanglement is generated. If only $\lambda_{3}=0$ and $\lambda_{2}>0$, the third qubit is decoupled from the bath and remains in $|+\rangle$ while the first two qubits follow the well-known two-qubit behavior [10]: For generic $\lambda_{2}$ and $f \rightarrow \infty$ the state of the first two qubits is completely mixed and separable states are reached for finite $f$, while in the symmetric case $\lambda_{2}=1$ the final state of the first two qubits is the separable mixture

$$
\rho_{2 s}=\frac{1}{2} \pi_{\psi^{+}}+\frac{1}{4}\left(\pi_{00}+\pi_{11}\right),
$$

with $\left|\psi^{+}\right\rangle=\frac{1}{\sqrt{2}}(|01\rangle+|10\rangle)$.

Special cases arise for all $\lambda_{j} \neq 0$ if $\lambda_{2}=\lambda_{3}, \lambda_{2}+\lambda_{3}=$ 1 , or $\lambda_{2}=1$. In the first case, the eigenvalues for the states $|001\rangle$ and $|010\rangle$ as well as those for $|101\rangle$ and $|110\rangle$ coincide. For $\lambda_{2}+\lambda_{3}=1$, the states $|011\rangle$ and $|100\rangle$ have the same eigenvalues. Finally, in the case $\lambda_{2}=1$ there is a degeneracy for the states $|010\rangle$ and $|100\rangle$ as well as for the states $|011\rangle$ and $|101\rangle$. Not all of the three relations above can be fulfilled at the same time. Moreover, $\lambda_{2}=1, \lambda_{3} \neq 1$ is equivalent up
TABLE I. The four special cases for $\rho(f \rightarrow \infty)$ depending on the parameters $\lambda_{2}, \lambda_{3}$, and the generic case where $\left(\lambda_{2}, \lambda_{3}\right)$ differs from the values in the special cases. The states used in the decompositions are $|W\rangle=\frac{1}{\sqrt{3}}(|001\rangle+|010\rangle+|100\rangle),|\bar{W}\rangle=$ $\frac{1}{\sqrt{3}}(|110\rangle+|101\rangle+|011\rangle)$, and $\left|\mathrm{GHZ}_{3}^{+}\right\rangle=\frac{1}{\sqrt{2}}(|011\rangle+|100\rangle)$. All asymptotic states are separable.

\begin{tabular}{lc}
\hline \hline Parameters & Asymptotic state $\rho(f \rightarrow \infty)$ \\
\hline$\lambda_{2}+\lambda_{3}=1$ & $\frac{1}{4} \pi_{\mathrm{GHZ}_{3}^{+}}+\frac{1}{8}\left(\pi_{000}+\pi_{001}+\pi_{010}+\pi_{101}+\pi_{110}+\pi_{111}\right)$ \\
$\lambda_{j} \notin\left\{0, \frac{1}{2}, 1\right\}$ & $\frac{1}{8} \pi_{000}+\frac{3}{8} \pi_{W}+\frac{3}{8} \pi_{\bar{W}}+\frac{1}{8} \pi_{111}$ \\
$\lambda_{2}=\lambda_{3}=1$ & $\frac{1}{4}\left(\pi_{\mathrm{GHZ}_{3}^{+}}+\pi_{0 \psi^{+}}+\pi_{1 \psi^{+}}\right)+\frac{1}{8}\left(\pi_{000}+\pi_{111}\right)$ \\
$\lambda_{2}=\lambda_{3}=\frac{1}{2}$ & $\frac{1}{2}\left(\pi_{0}+\pi_{1}\right) \otimes \rho_{2 s}$ \\
$\lambda_{2}=\lambda_{3}$ & $\frac{1}{8} \mathbb{1} \otimes \mathbb{1} \otimes \mathbb{1}$ \\
$\notin\left\{0, \frac{1}{2}, 1\right\}$ & \\
Otherwise & \\
\hline \hline
\end{tabular}

to rescaling to $\lambda_{2}=\lambda_{3} \neq 1$ after exchanging the first with the third qubit. Finally, if the second and third equalities are fulfilled, we have $\lambda_{3}=0$ which decouples the third qubit. Hence there remain four (truly tripartite) special cases with the corresponding asymptotic states $\rho(f \rightarrow \infty)$, which are located at the border between separable and biseparable states; see Table I. The negativity is nonzero for $t \rightarrow \infty$ for at least one bipartition in each of those special cases (cf. Fig. 3).

By using Eq. (5) an explicit form of the effective interaction can be obtained from [36,37]

$$
H_{\mathrm{eff}}=-\sum_{j} \frac{g_{j}^{2}}{4 \omega_{j}} S^{2} .
$$

The mixed terms in the expansion of $S^{2}$ in terms of singleparticle spin operators show that the heat bath induces an effective Ising interaction, and it is known that with such an interaction a GHZ state can be generated from the state $|+++\rangle$ [38]. The imaginary part of the bath correlation
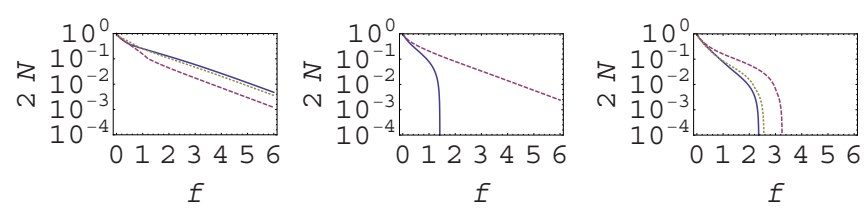

FIG. 3. (Color online) Negativities $2 \mathcal{N}(f)$ in the asymptotic states according to Table 1 for values of $\varphi$ maximizing $\mathcal{N}(f=0)$. In each plot, the blue solid line gives the negativity for the bipartition $\{1 \mid 23\}$, the red long-dashed line for the bipartition $\{2 \mid 13\}$, and the green dashed line for the bipartition $\{3 \mid 12\}$. (a) For $\lambda_{2}+\lambda_{3}=1$ (first line of Table 1) the negativities decay exponentially, with a specific decay constant for each qubit. We do not show examples for the second and the third lines of Table 1, since the only difference with the first line is that all qubits have the same decay constant $\left(\lambda_{2}=\lambda_{3}=1\right)$, or only the decay constant of the first qubit differs from the others $\left(\lambda_{2}=\lambda_{3}=\frac{1}{2}\right)$. The plot shows the negativities for $\lambda_{2}=2 / 3, \lambda_{3}=1 / 3$ and $\varphi=3 \pi / 8$. (b) For $\lambda_{2}=\lambda_{3} \notin\left\{0, \frac{1}{2}, 1\right\}$ the negativity of the first qubit vanishes at a finite value of $f$ while that of the other qubits vanishes smoothly. Plot parameters are $\lambda_{2}=\lambda_{3}=1 / 3$ and $\varphi=3 \pi / 8$. (c) In the generic case all negativities vanish at finite values of $f$ (here for $\lambda_{2}=\pi / 4, \lambda_{3}=e / 4$ and $\varphi=1 / 2$ ). 
function directly reflects the influence of this effective interaction [see Eq. (3)].

In applications also the distance dependence of the effective interaction is of interest. For this purpose, of course, a realistic model of a specific physical systems has to be studied. In Ref. [39] it was found that, in the case of two harmonic oscillators coupled to a common heat bath of other oscillators, entanglement is generated only over a small distance. However, that model contains dissipation in addition to the dephasing studied in the present paper. For a study of the distance dependence of the entanglement generation one should therefore rather compare with Ref. [36], where quantum dots with degenerate energy levels were coupled to blackbody radiation, and it was found that for large-enough times bipartite entanglement can spread over macroscopic distances. Since the same bath correlations enter the entanglement dynamics for both types of systems, we expect that similar statements as in Ref. [36] concerning the distance and time dependence of the created entanglement also apply to the multipartite case.

\section{SUMMARY}

We have analyzed multipartite entanglement generation in an exactly solvable dephasing model of three noninteracting qubits coupled to the same thermal heat bath. Entanglement generation solely depends on the interplay between the real and imaginary parts $f(t)$ and $\varphi(t)$ of the bath-correlation function, and two parameters $\lambda_{2}, \lambda_{3}$ of the coupling Hamiltonian.

By using optimized lower bounds to the three tangle and the GME concurrence, we have shown that, for small $f(t)$ (weak decoherence) and sufficiently large $\varphi(t)$ (strong effective interaction mediated by the heat bath), all classes of tripartite entanglement can be generated from almost any pure product state. For almost all pairs $\left(\lambda_{2}, \lambda_{3}\right)$ the state for sufficiently large finite $f$ is fully separable, but there are four special symmetric cases, where the reservoir-induced bipartite entanglement is proven to persist up to arbitrarily large values of $f(t)$. If the bath correlation function $f(t)$ for $t \rightarrow \infty$ saturates at sufficiently small values, genuine tripartite and even GHZ-type entanglement may be present up to arbitrarily large times. One may note that these statements hold as well for weakly mixed initial separable states.

While our work is intended as a theoretical proof of principle, one may nevertheless wonder in what kind of systems one could observe the predicted effects. The starting point of degenerate energy levels suggest using systems with strong couplings to the environment compared to the energy splittings of the individual systems. Circuit or cavity quantum electrodynamics systems, or quantum dots with large dipole matrix elements in small cavities, respectively, might offer such a possibility. Heat baths with essentially monochromatic modes, such as optical phonons, in resonance with three spins could be another option. There, the system Hamiltonian can be eliminated by working in the interaction picture, and due to the resonance with all modes the interaction Hamiltonian still remains time independent. Couplings can be controlled in these systems by the mode amplitudes of the electromagnetic field, and dipole matrix elements of the embedded quantum systems.

\section{ACKNOWLEDGMENTS}

This work was funded by the German Research Foundation within SPP 1386 (C.E.), by the Basque Government Grant No. IT-472-10 and MINECO Grant No. FIS2012-36673-C0301 (J.S.). C.E. and J.S. thank J. Fabian and K. Richter for their support.
[1] R. Horodecki, P. Horodecki, M. Horodecki, and K. Horodecki, Rev. Mod. Phys. 81, 865 (2009).

[2] M. B. Plenio, S. F. Huelga, A. Beige, and P. L. Knight, Phys. Rev. A 59, 2468 (1999).

[3] A. Beige, D. Braun, and P. L. Knight, New J. Phys. 2, 22 (2000).

[4] A. Beige, D. Braun, B. Tregenna, and P. L. Knight, Phys. Rev. Lett. 85, 1762 (2000).

[5] S. Schneider and G. J. Milburn, Phys. Rev. A 65, 042107 (2002).

[6] B. Kraus, H. P. Büchler, S. Diehl, A. Kantian, A. Micheli, and P. Zoller, Phys. Rev. A 78, 042307 (2008).

[7] F. Verstraete, M. M. Wolf, and J. I. Cirac, Nat. Phys. 5, 633 (2009).

[8] Y. Lin, J. P. Gaebler, F. Reiter, T. R. Tan, R. Bowler, A. S. Sørensen, D. Leibfried, and D. J. Wineland, Nature (London) 504, 415 (2013).

[9] S. Shankar, M. Hatridge, Z. Leghtas, K. M. Sliwa, A. Narla, U. Vool, S. M. Girvin, L. Frunzio, M. Mirrahimi, and M. H. Devoret, Nature (London) 504, 419 (2013).

[10] D. Braun, Phys. Rev. Lett. 89, 277901 (2002).

[11] M. S. Kim, J. Lee, D. Ahn, and P. L. Knight, Phys. Rev. A 65, 040101(R) (2002).
[12] F. Benatti, R. Floreanini, and M. Piani, Phys. Rev. Lett. 91, 070402 (2003).

[13] F. Benatti and R. Floreanini, Phys. Rev. A 70, 012112 (2004).

[14] F. Benatti and R. Floreanini, Int. J. Mod. Phys. B 19, 3063 (2005).

[15] F. Benatti, A. M. Liguori, and A. Nagy, J. Math. Phys. 49, 042103 (2008).

[16] J. P. Paz and A. J. Roncaglia, Phys. Rev. Lett. 100, 220401 (2008).

[17] S. Maniscalco, F. Francica, R. L. Zaffino, N. Lo Gullo, and F. Plastina, Phys. Rev. Lett. 100, 090503 (2008).

[18] F. Francica, S. Maniscalco, J. Piilo, F. Plastina, and K.-A. Suominen, Phys. Rev. A 79, 032310 (2009).

[19] L. Huai-Xin, Chin. Phys. 16, 1878 (2007).

[20] J.-H. An, S.-J. Wang, and H.-G. Luo, Phys. A 382, 753 (2007).

[21] G.-X. Li, L.-H. Sun, and Z. Ficek, J. Phys. B: At., Mol. Opt. Phys. 43, 135501 (2010).

[22] F. Benatti and A. Nagy, Ann. Phys. (NY) 326, 740 (2011).

[23] N. Pumulo, I. Sinayskiy, and F. Petruccione, Phys. Lett. A 375, 3157 (2011).

[24] W. Dür, G. Vidal, and J. I. Cirac, Phys. Rev. A 62, 062314 (2000). 
[25] A. Acin, D. Bruß, M. Lewenstein, and A. Sanpera, Phys. Rev. Lett. 87, 040401 (2001).

[26] A. Borras, A. P. Majtey, A. R. Plastino, M. Casas, and A. Plastino, Phys. Rev. A 79, 022108 (2009).

[27] C. Eltschka and J. Siewert, arXiv:1402.6710 [J. Phys. A: Math. Gen. (to be published)].

[28] D. Braun, F. Haake, and W. T. Strunz, Phys. Rev. Lett. 86, 2913 (2001).

[29] V. Coffman, J. Kundu, and W. T. Wootters, Phys. Rev. A 61, 052306 (2000).

[30] O. Viehmann, C. Eltschka, and J. Siewert, Appl. Phys. B 106, 533 (2012)

[31] A. Uhlmann, Open Sys. Info. Dyn. 5, 209 (1998).

[32] C. Eltschka and J. Siewert, Sci. Rep. 2, 942 (2012).
[33] Z.-H. Ma, Z.-H. Chen, J.-L. Chen, C. Spengler, A. Gabriel, and M. Huber, Phys. Rev. A 83, 062325 (2011).

[34] G. Vidal and R. F. Werner, Phys. Rev. A 65, 032314 (2002).

[35] S. L. Braunstein, C. M. Caves, R. Jozsa, N. Linden, S. Popescu, and R. Schack, Phys. Rev. Lett. 83, 1054 (1999).

[36] D. Braun, Phys. Rev. A 72, 062324 (2005).

[37] G. D. Mahan, Many-Particle Physics (Kluwer Academic/ Plenum Publishers, New York, 1990).

[38] M. Hein, W. Dür, J. Eisert, R. Raussendorf, M. V. den Nest, and H.-J. Briegel, Entanglement in Graph States and its Applications, Proceedings of the International School of Physics “Enrico Fermi” (IOS Press, Amsterdam, 2006), Vol. 162, p. 115.

[39] T. Zell, F. Queisser, and R. Klesse, Phys. Rev. Lett. 102, 160501 (2009). 